\title{
Research Paper \\ Addiction Potential Based-on Atypical Anxious-Impulsive Pattern of Social Anxiety: The Role of Anger and Impulsivity
}

${ }^{*}$ Tooraj Sepahvand ${ }^{1}$

1. Assistant Professor, Department of Psychology and Educational Sciences, Faculty of Humanities, Arak University, Arak, Iran.

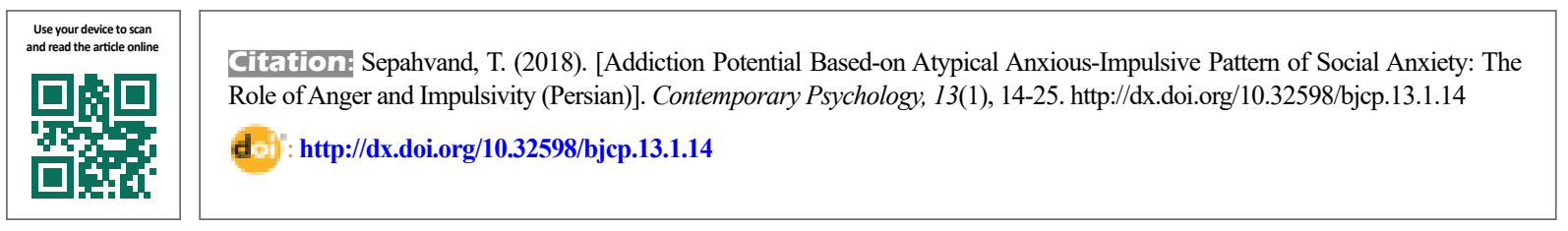

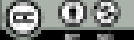

Received: 13 Sep 2017 Accepted: 10 Feb 2018 Available Online: 21 Mar 2018

Key words: Social anxiety, Atypical anxious-impulsive pattern, Addiction potential, Anger

\section{A B STRACT}

Objectives The present research aimed to study the addiction potential in social anxiety based on atypical anxious-impulsive pattern of this disorder (with regard to anger and impulsivity).

Methods This research was carried out with correlational design and predictive capability. For this purpose, among the students of Arak University, a sample of 470 students were selected based on convenience sampling method. At first, the Connor Social Phobia Inventory (SPIN), Zargar addiction potential, Novaco anger and Dickman impulsivity scales were administered to them. After the assessment of the results, 119 participants who had social anxiety (scores 19 and more) and their lie scale of addiction potential was without any problem, were recruited for the study.

Results Analysis by simultaneous multiple regression indicated that anger and impulsivity predicted almost $38 \%$ of variance of addiction potential in individuals with social anxiety $(F=35.395 ; P<0.001)$, and impulsivity had significant role in this prediction $(\beta=0.542)$, while the anger did not have any significant role.

Conclusion In conclusion, impulsivity is a very important variable that according to its atypical anxiousimpulsive pattern of social anxiety, it is necessary to be seriously considered in understanding addiction potential of individuals with social anxiety and in reduction of pathologies related to them. 


\section{آمادكَى اعتياد بر مبناى الكَّى غيرمعمول مضطربتكانشكَ اضطراب اجتماعى: نقش خشم و تكانشَّرى}

• تورج سيهوند' 1- استاديار، كروه روانشناسى و علوم تربيتى، دانشكده علوم انسانى، دانشعاه اراك، اراك، ايران.

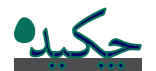

هدف اين يُروهش تبيين آمادكى اعتياد در اضطراب اجتماعي بر مبناى الكوى غيرمعمول مضطربتكانشكر اين اختلال (با توجه به نقش خشم

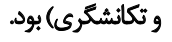

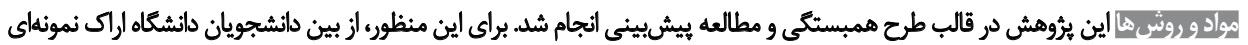

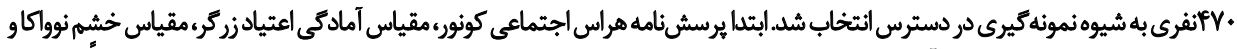

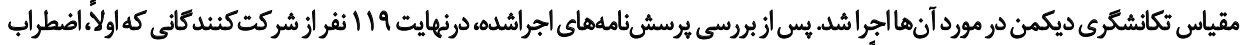

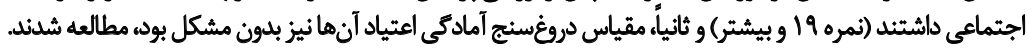

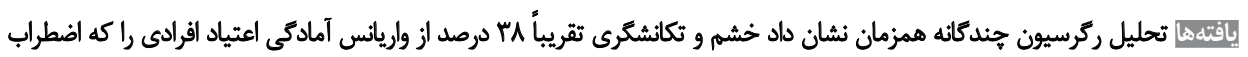

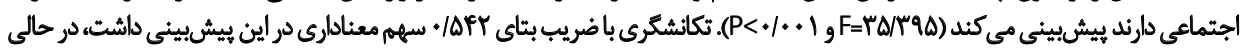

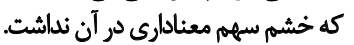

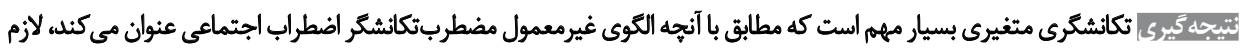

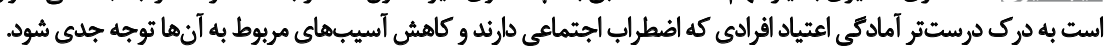

تاريخ دريافت: rا شهريور Ir99

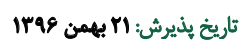

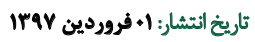

كليدوارهها:

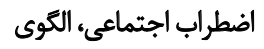

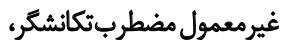

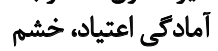

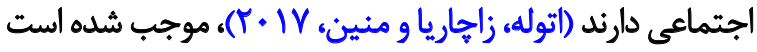

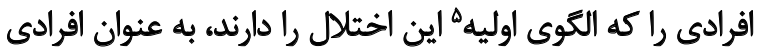

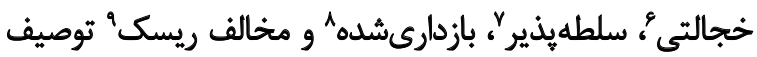

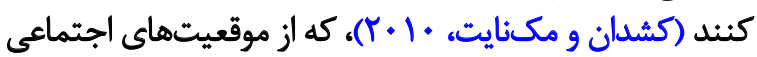

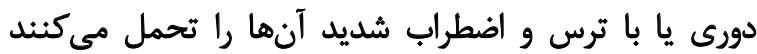

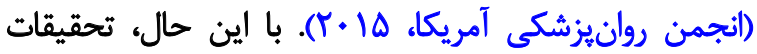

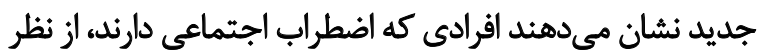
كيفي واكنشها و راهبردهاى نظمبخشيى متفاونى

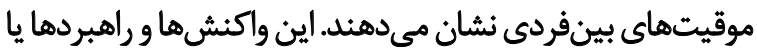

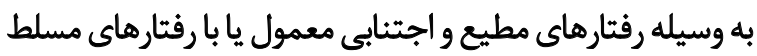

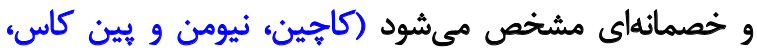

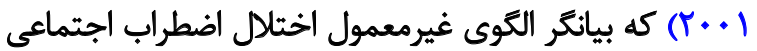

5. Prototypical person

6. Shy

7. Submissive

8. Inhibited

9. Risk averse dale

اضطراب اجتماعى 'كه به عنوان هراس اجتماعى بنيز شناخته

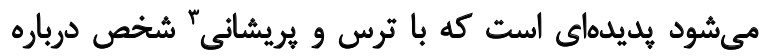

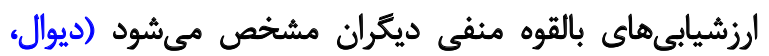

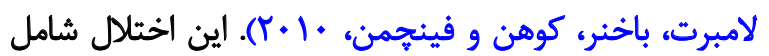

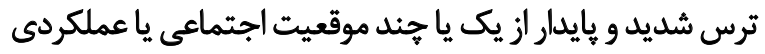

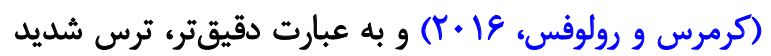

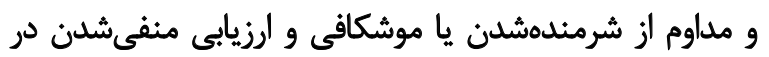

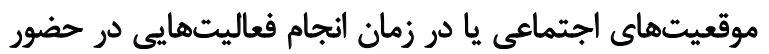

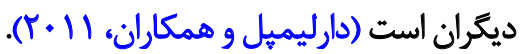
ظاهراً تأكيد بر اجتناب" زياد در افرادى كه اختلال اضطراب
1. Social anxiety
2. Social phobia
3. Distress
4. Avoidance 


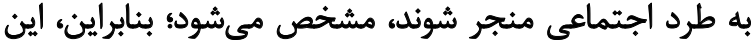

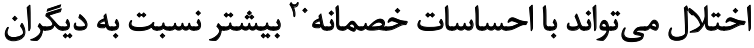

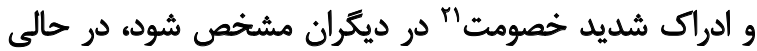

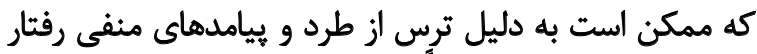

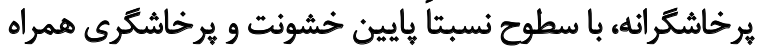

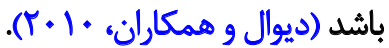

با وجود جنين تصوراتى، تعدادى از محققان كزارش كردهاند

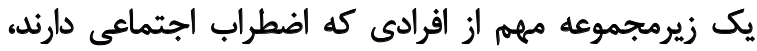

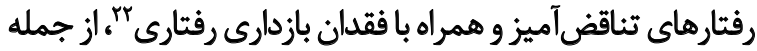

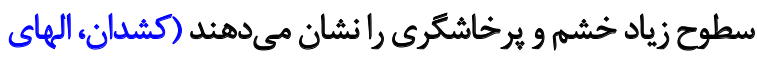

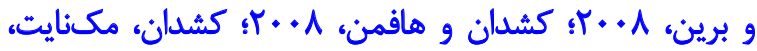

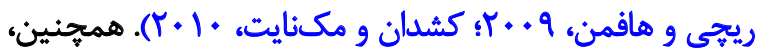

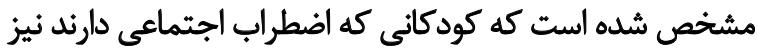

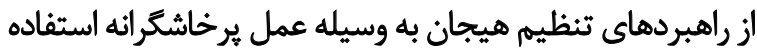

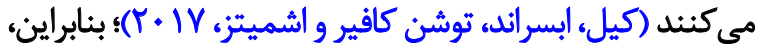

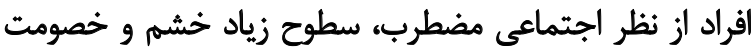

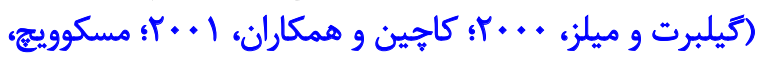

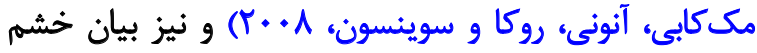

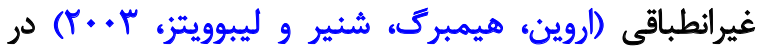

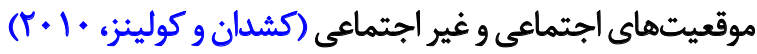
دارند.

به نظر مىرسد خشم افرادى كه اضطراب اجتماعى دارند

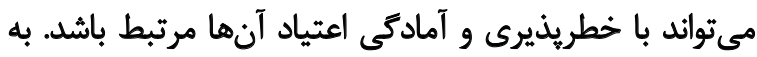

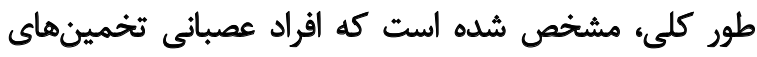

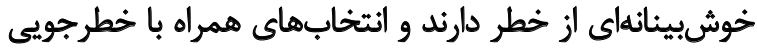

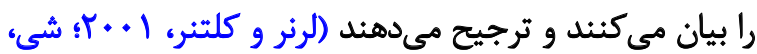

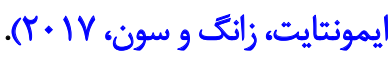

اين خطريذيرى زياد در افرادى كه اضطراب اجتماعى دارنده با

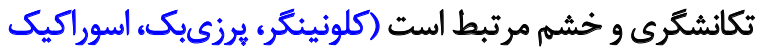

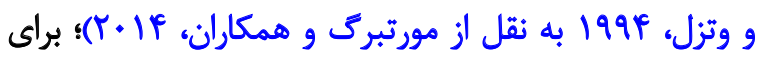

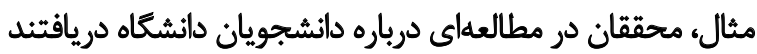

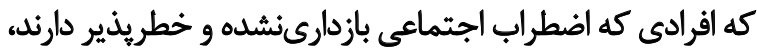

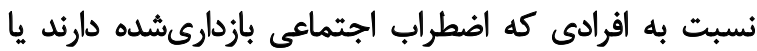

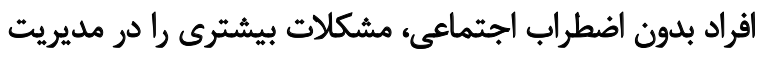

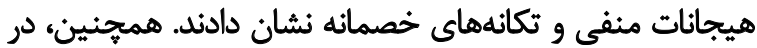

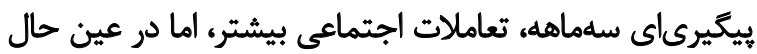

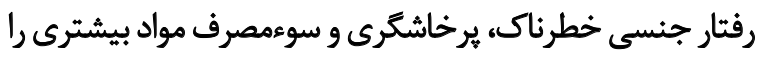

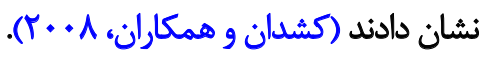
يكى ديكر از متغيرهايي كه در تمايل به اعتياد افرادى كه

20. Hostile feelings

21. Heightened perceptions of hostility

22. Dehavioral disinhibition

$$
\text { هستند (مورتبر تى، تيلفورث، ونزالك وكر، IF) IF). }
$$

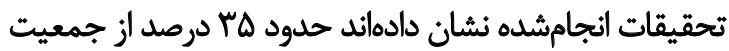

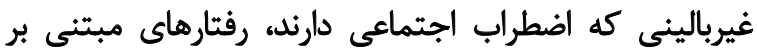

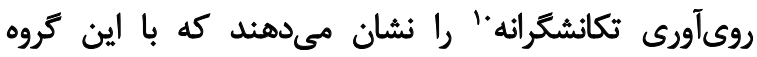

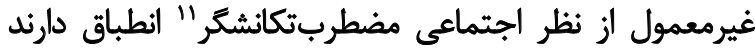

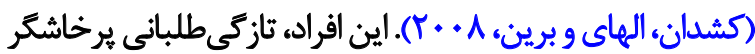

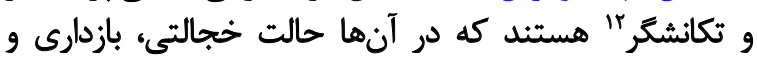

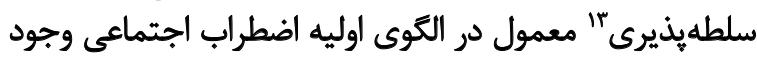

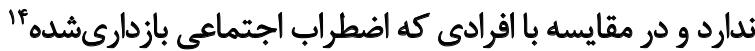

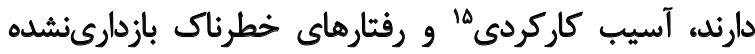

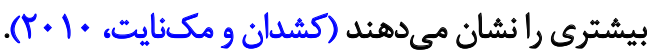
به عبارتى، اين زيرتروه از افراد كه اضطراب اجتماعى

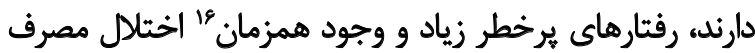

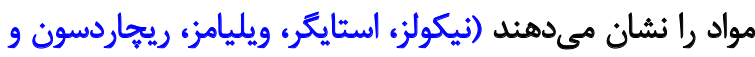

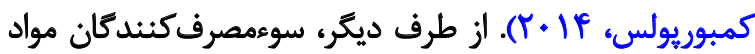

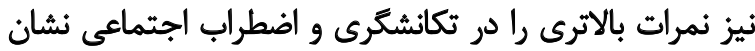

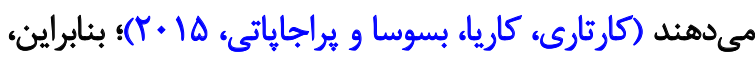

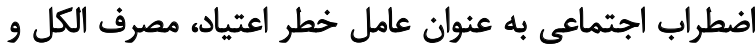

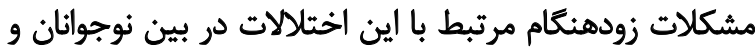

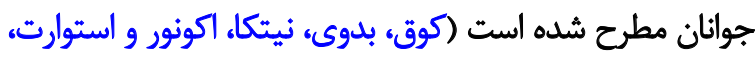

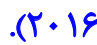

مسئله اعتياد نتيجه فرايندى است كه در آن عوامل متعددى دري

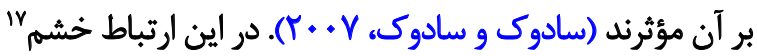

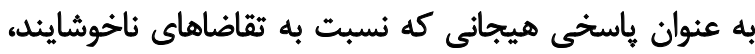

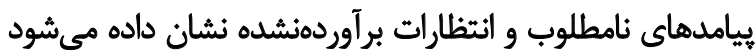

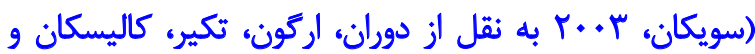

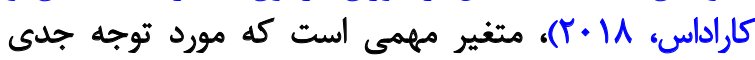

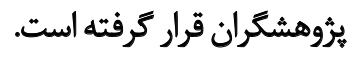

يافتههاى مختلفى وجود خشم و خصومت را در افرادى كه

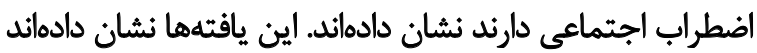

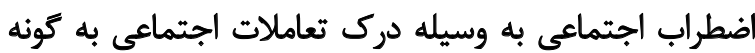

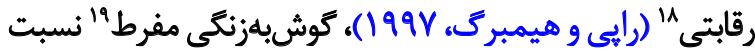

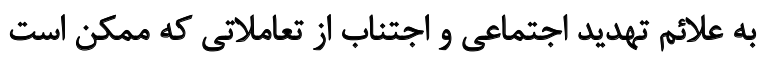

10. Approach-based impulsive behaviors

11. Atypical socially anxious-impulsive group

12. Aggressive, impulsive novelty seekers

13. Submissiveness

14. Inhibited socially anxious

15. Functional impairment

16. Co-morbidity

17. Anger

18. Competitive

19. Hypervigilance 


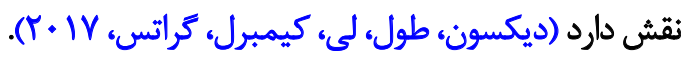

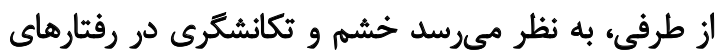

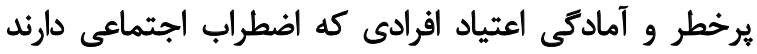

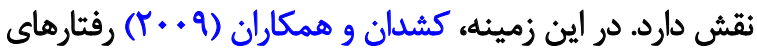

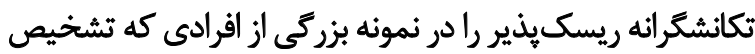

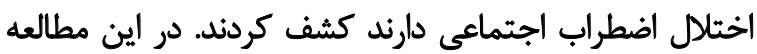

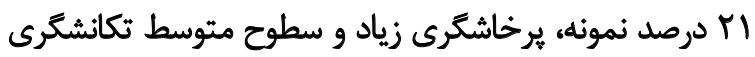

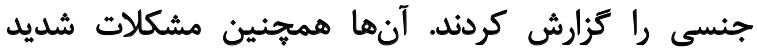

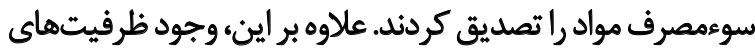

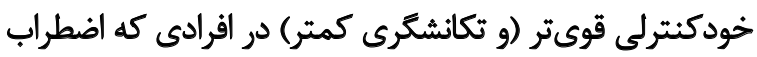

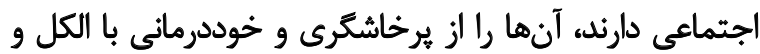

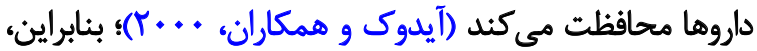

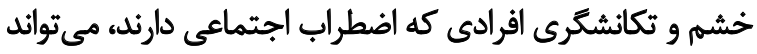

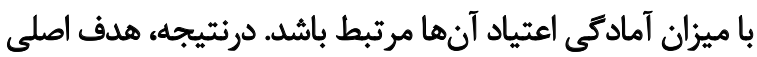

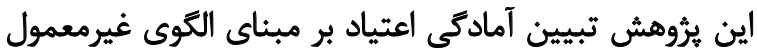

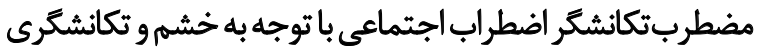

og.

(ُ)

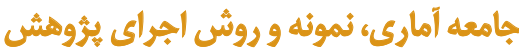

براى انجام اين يُروهش از طرح همبستخى و مطالعه

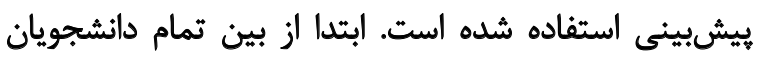

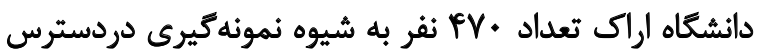

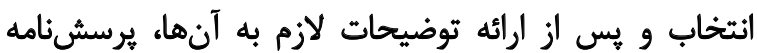

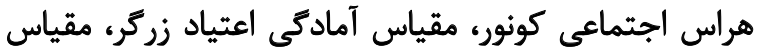

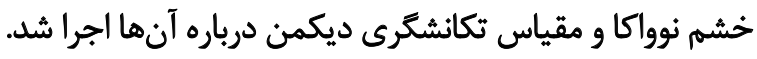

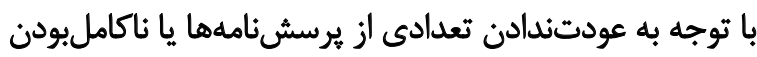

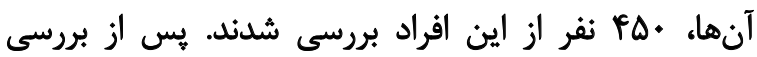

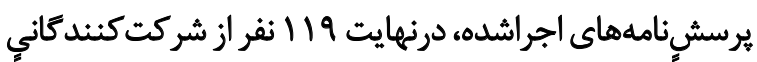

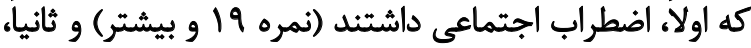

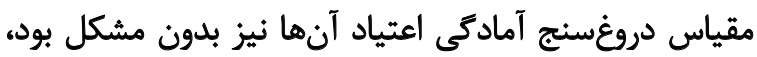

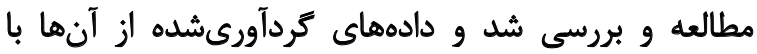
استفاده از ركرسيون جندكانه همزمان تحليل شداد

إبزإ)

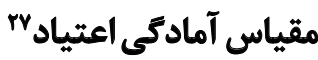

ويد و بوجر^ج (1991) مقياس آمادكى اعتياد را ساختهاند.

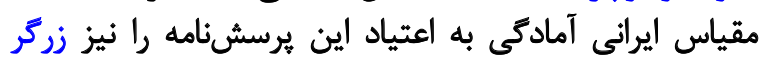

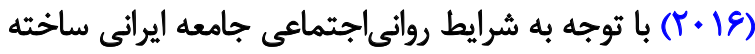

27. Addiction Potential Scale

28. Weed \& Butcher

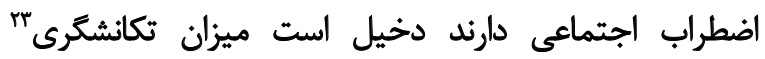

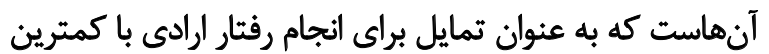

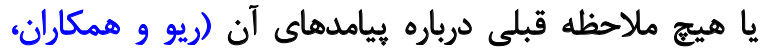

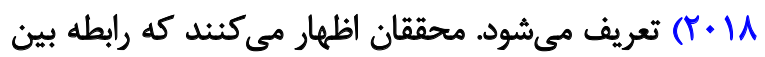

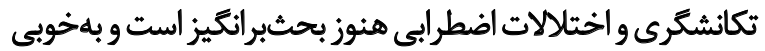

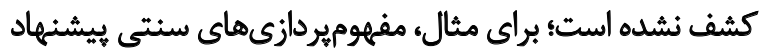

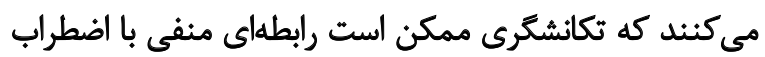

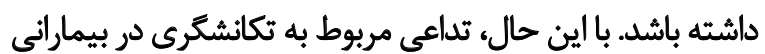

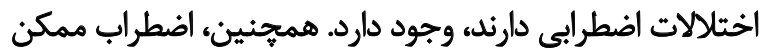

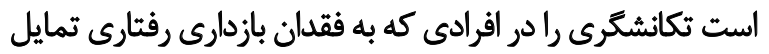

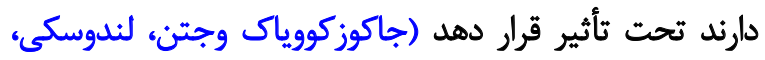

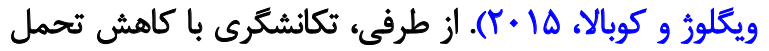

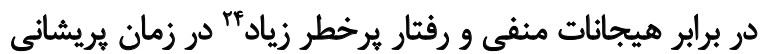

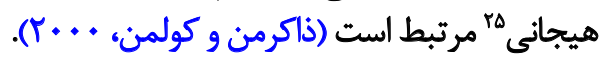

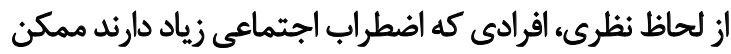

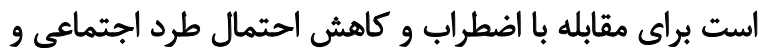

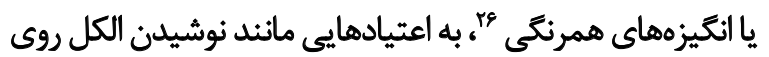

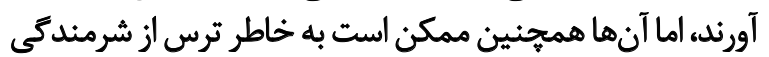

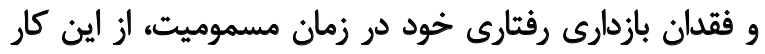

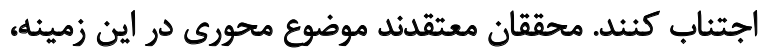

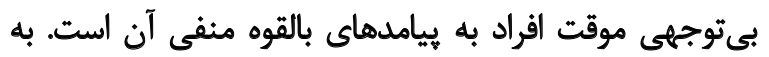

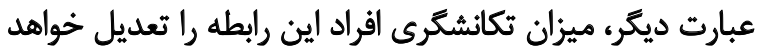

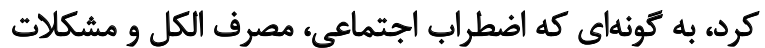

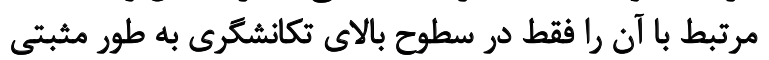

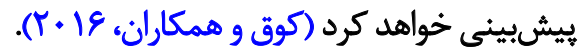

محققان ديكر شواهدى يافتهاند مبنى بر اينكه افرادى كه

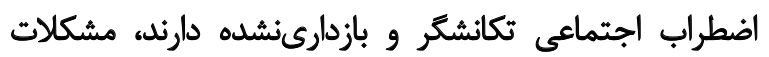

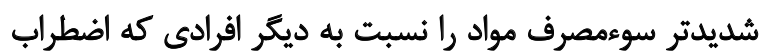

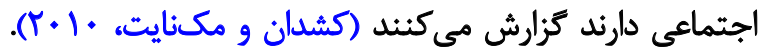

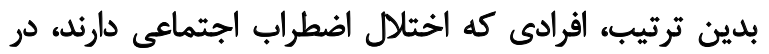

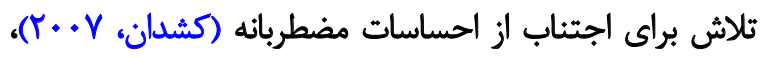

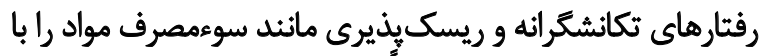

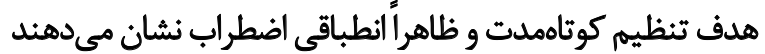

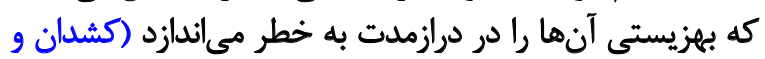

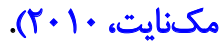

درمجموع، به نظر مىرسد تكانشكَرى و خشم در افرادى كه

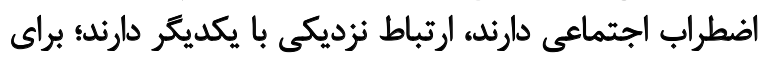

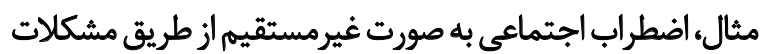
كنترل تكانه ايجادشده به وسيله هيجان، در خشمه و يرخاشكرى إنى

23. Impulsivity

24. Increased risky behavior

25. Emotionally distressed

26. Conformity motives 


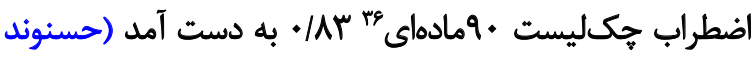

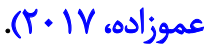

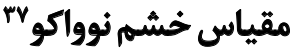

مقياس خشم نوواكو (1999) يك ابزار خود خُزارشى است

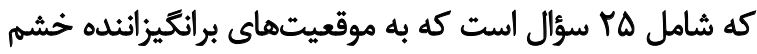

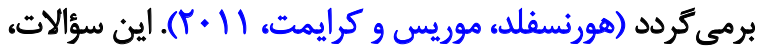

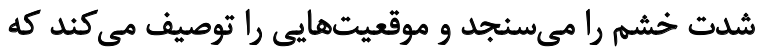

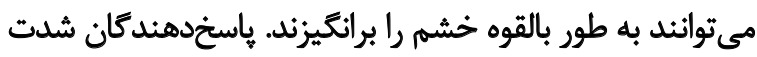

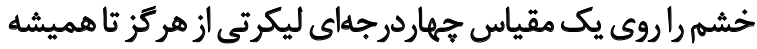

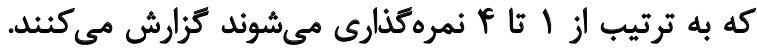

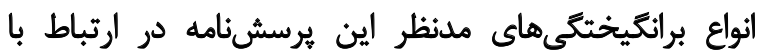

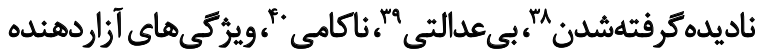

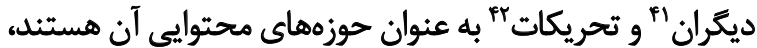

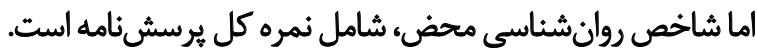

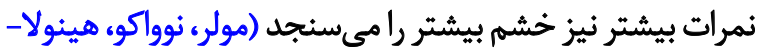

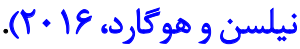

هايايي و روايى اين آزمون را محققان مختلف ثأييد كرداند

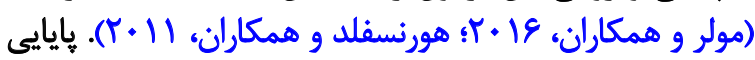

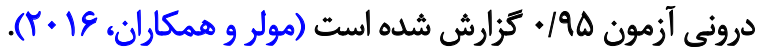

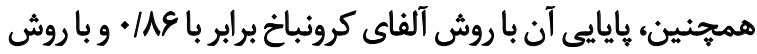

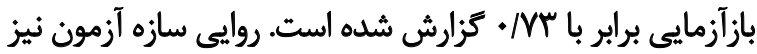

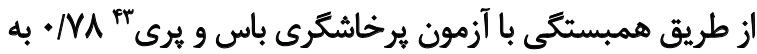

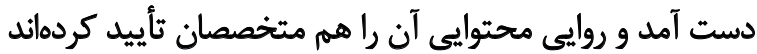

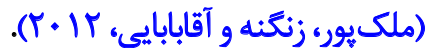

\section{مقياس تكانشكَرى ديكمن}

اين مقياس را (ديكمن، • 199) ساخته است و مب سؤال دارد.

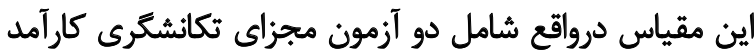

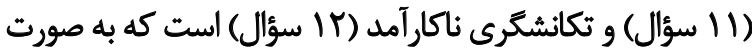

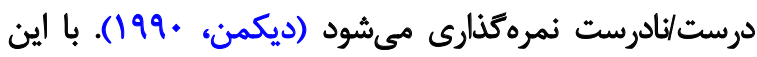

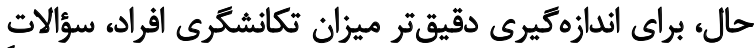

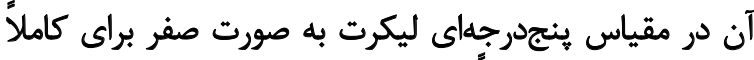

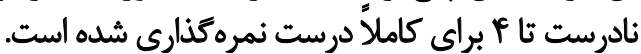

36. Symptom Checklist-90

37. Novaco Anger Scale

38. Disrespect

39. Unfairness

40. Frustration

41. Annoying traits of others

42. Irritations

43. Buss \& Perry

44. Dickman Impulsivity Scale
است. اين يرسش نامه \&ب ماده به اضافه ينج ماده دروغسنج (مواد

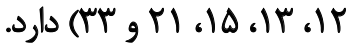

نمره كذارى هر سؤال به صورت ليكرتى از صفر (كاملأمخالفم) تا نا

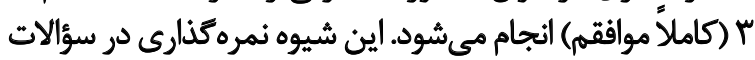

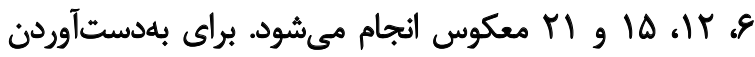

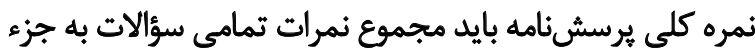

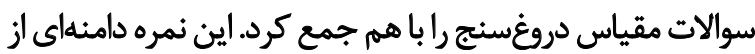

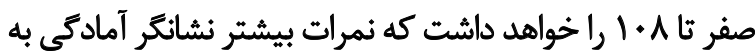
اعتياد بيشتر در فرد است و برعكس.

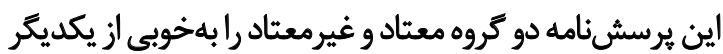

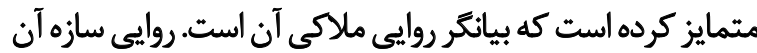

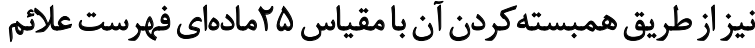

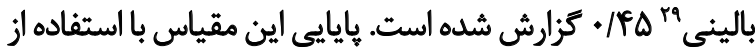

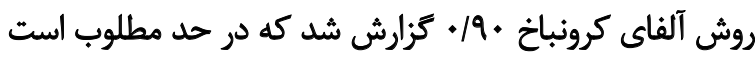

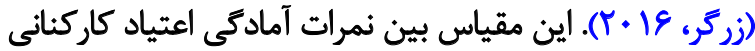

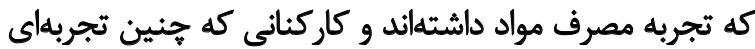

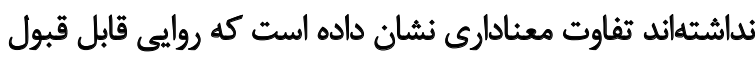

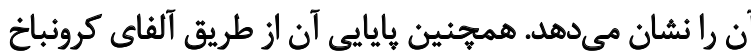

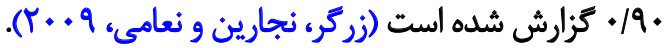

\section{برسش نامه هراس اجتماعى}

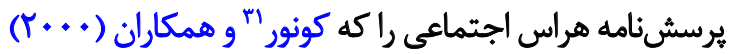

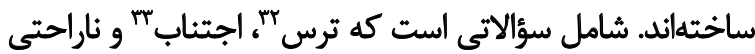

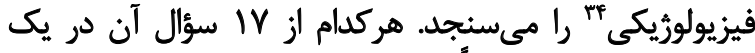

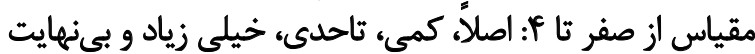

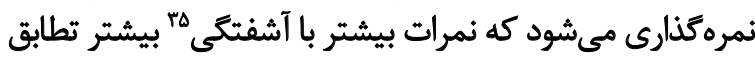
دارد. به اين ترثيب، نمره كل مقياس ازئ صفر ثا بـ 9 است.

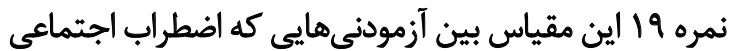

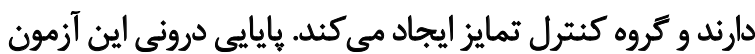

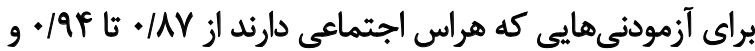

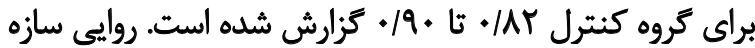

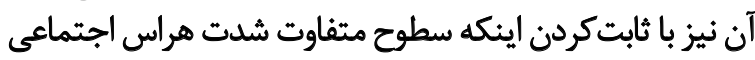

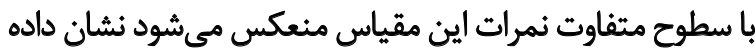

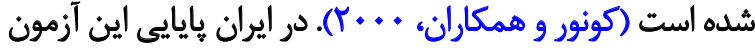

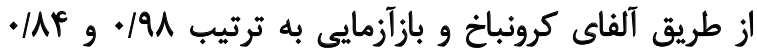

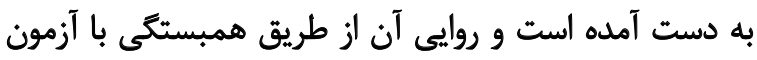

29. Symptoms Checklist-25 (SCL-25)

30. Social Phobia Inventory (SPIN)

31. Connor

32. Fear

33. Avoidance

34. Physiological discomfort

35. Distress 
جدول ا. ميانكين و انحراف استاندارد متغيرهاي سن و اضطراب اجتماعى

\begin{tabular}{|c|c|c|}
\hline انحراف استاندارد & مياتكين & متغير \\
\hline IVE & $r / / r$ & سن \\
\hline $1.1 . \mathrm{V}$ & $M r / 1$ & اضطراب اجتماعي \\
\hline
\end{tabular}

جدول I. ميانكين و انحراف استاندارد متغيرهاي آمادكى اعتياد، خشمه و نكانشكرى

\begin{tabular}{|c|c|c|}
\hline انحراف استاندارد & مياتكين & مثغير \\
\hline $11 / n$ & PNAY & آمادكى اعتياد \\
\hline $9 / 10$ & $8 \mathrm{Q} / \cdot A$ & خشم \\
\hline NII & $\Delta N)_{1}$ & تكانشكرى \\
\hline
\end{tabular}

دوفصلنامه روانشناسى معاصر

جدول "ا. همبستكى متغيرهاى آمادكى اعتياد، خشمه و تكانشكُى با يكديكً

\begin{tabular}{|c|c|c|c|}
\hline$r$ & $r$ & 1 & متغير \\
\hline & & 1 & آهادكى اعثياد \\
\hline & 1 & $. / 4)^{\circ}$ & خشم \\
\hline 1 &.$/ \Delta e^{*}$ & $.181^{\circ}$ & تكانشكرى \\
\hline
\end{tabular}

بررسى شده با ميانكين سنى rV/M سال و انحراف استائدارد

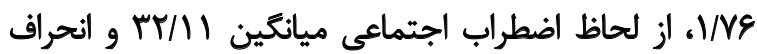

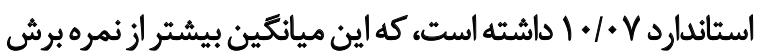

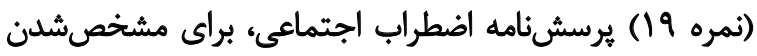
وجود اضطراب اجتماعى در اعضاى كروه نمونه است. در ادامه

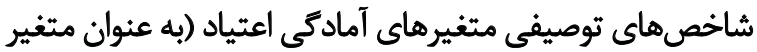

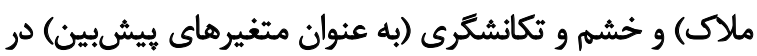

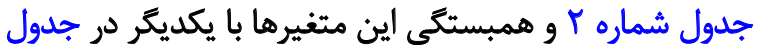

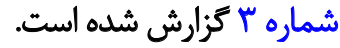

جدول شماره Y ميانكين و انحراف استاندارد متغيرهاي آمادكى

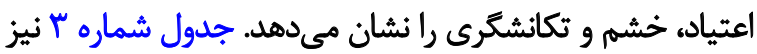

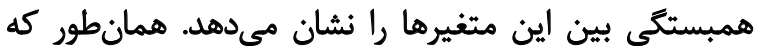

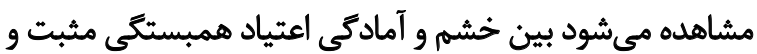

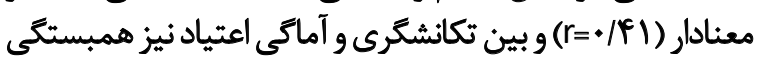

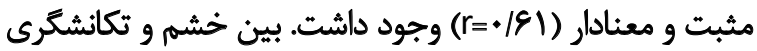

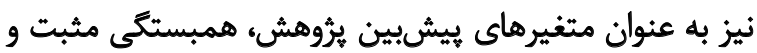

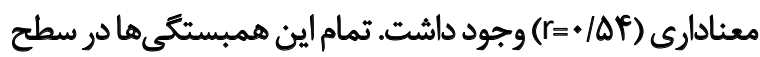

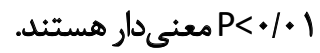

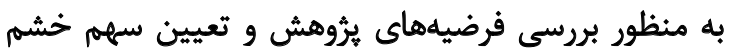

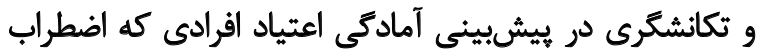

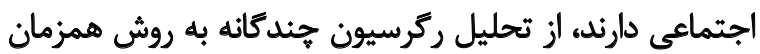

با معكوس كردن نمرهكذارى سؤالات مربوط به تكانشكرى

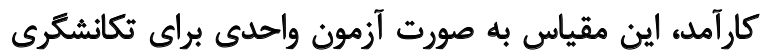

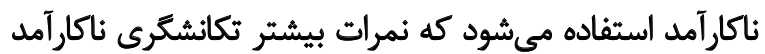

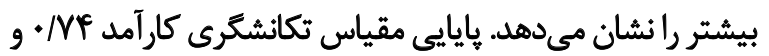

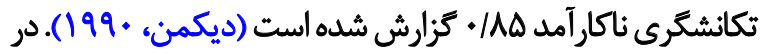

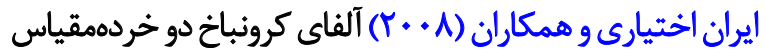

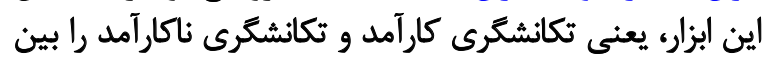

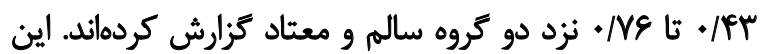
مقياس با ديكر مقياس هاي تكانشغرى مانئد مقياس تكانشكرى

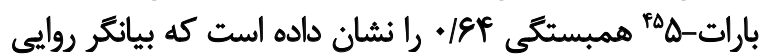
قابل قبول آن است (ديكمنه • 199). Lodịl

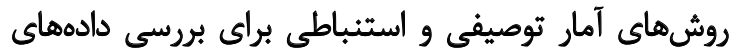

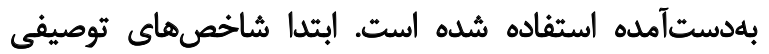
وضعيت سنى و اضطراب اجتماعى (به عنوان متغير كنترل)

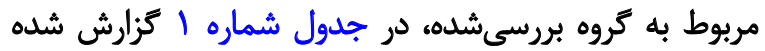

تحليلهاى توصيفى جدول شماره 1 نشان ميدهد گروه

45. Barratt Impulsivity Scale-5 
جدول F. خلاصه مدل و نتايج تحليل واريانس مربوط به ركرسيون بيشبينى آمادكى اعتياد بر مبناى خشم و تكانشكرى

\begin{tabular}{|c|c|c|c|c|c|c|c|}
\hline Sig. & $\mathbf{F}$ & ضريب تعيين & ضريب همبستكى & مياتكين مجذورات & درجات أزادى & مجموع مجذورات & مدل \\
\hline & & & & $r+9 \% / q 9 \Delta$ & $r$ & S1rv/4R & ركرسيون \\
\hline \multirow[t]{2}{*}{$\%$} & $r \Delta / \% q \Delta$ & . & .185 & $N A / D \& Q$ & 118 & $1 . .+1 / / 9 \Delta A$ & باقيمائده \\
\hline & & & & & 111 & IFIEQ/G9V & كل \\
\hline
\end{tabular}

جدول هـ ضرايب ركرسيون متغيرهاى بيشبين آمادكي اعثياد

\begin{tabular}{|c|c|c|c|c|c|c|}
\hline Sig. & $\mathbf{T}$ & ضرايب استانداردشده & خطاى استاندارد & ضرايب استانداردنشده & مدل & متغير ملاك \\
\hline$\cdot / r \cdot F$ & זr/1/ & & $1 / 4 \pi$ & $V / T V q$ & مقدار ثابت & \\
\hline .198 & $1 /$ rap &.$/ M$ &.$/ 111$ & $\cdot / 1 \Delta \Delta$ & خشم & آهادكى اعثياد \\
\hline$\%$ & s/rTq & . IAFT & 㑭 &.$/ N \lambda_{\text {. }}$ & تكانشكرى & \\
\hline
\end{tabular}

دوفصلنامه وزانشناسى معاصر

دارند ميزان تكانشكرى آنهاست.

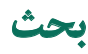

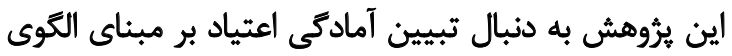

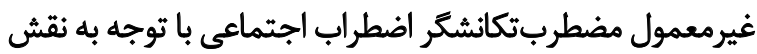

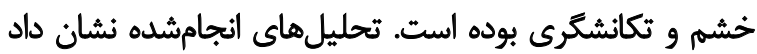

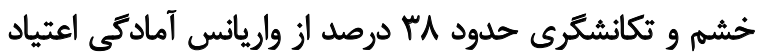

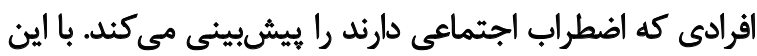

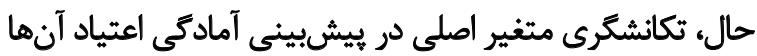

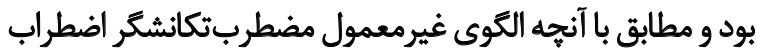

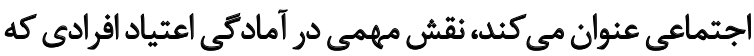

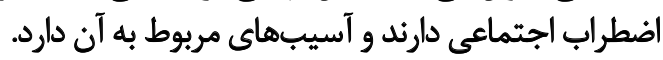

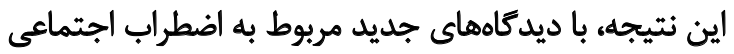

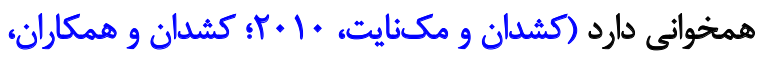

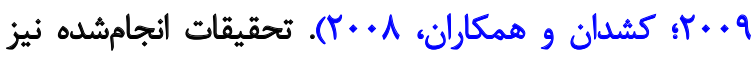

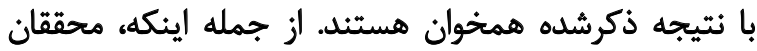

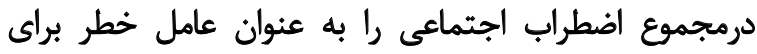

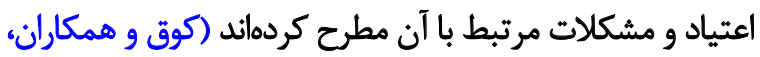

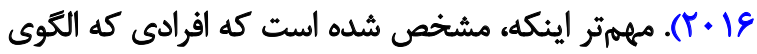
مضطربتكانشكر اضطراب اجتماعى دارنده آسيبهاي ماندي مختلف

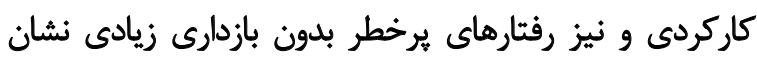

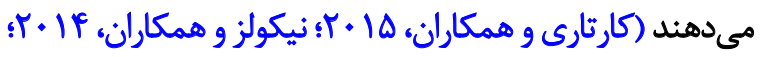

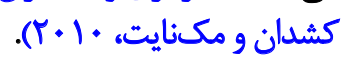

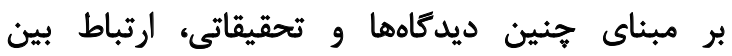

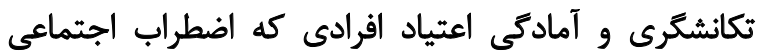

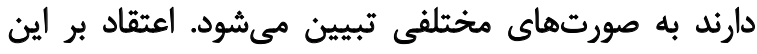

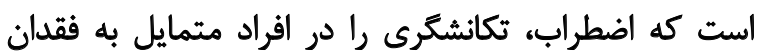

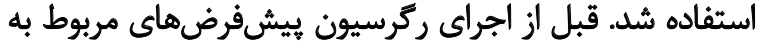

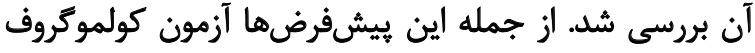

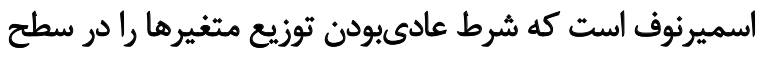

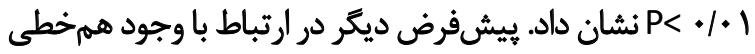

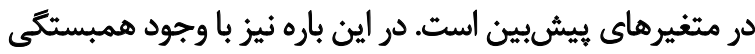

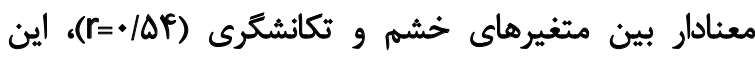

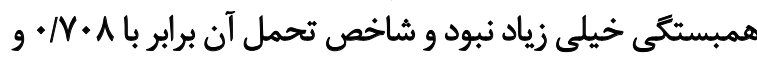

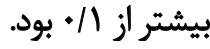

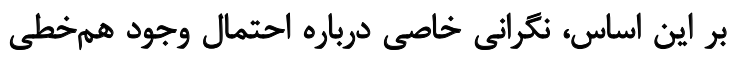

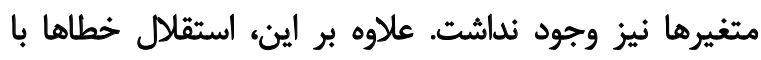

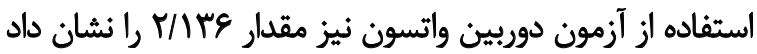

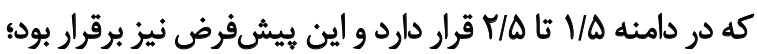

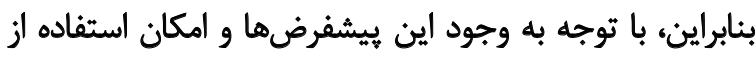

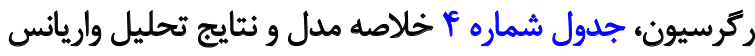

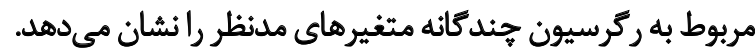

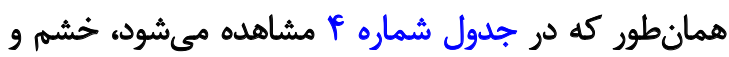

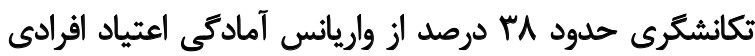

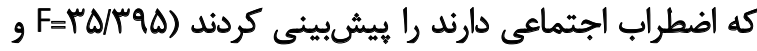

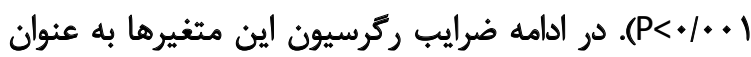

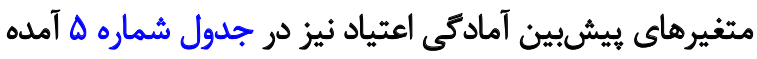
است. متغير

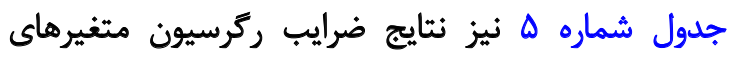

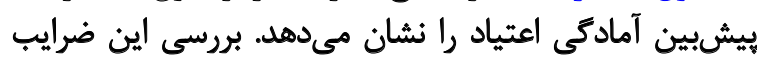

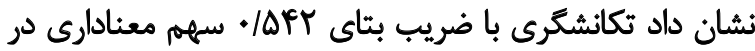

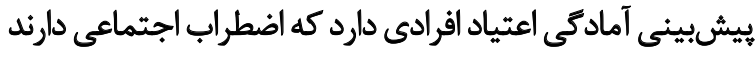

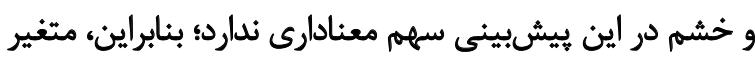

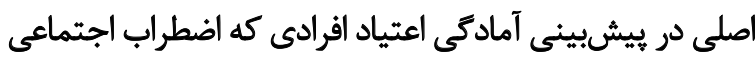


اجتماعى دارند نيز تبيينهاى جهندى وجود دارد. از جمله اينكه

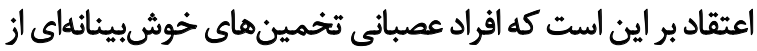

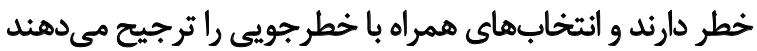

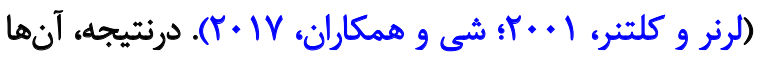

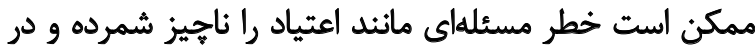

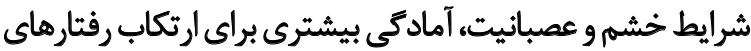

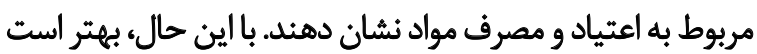

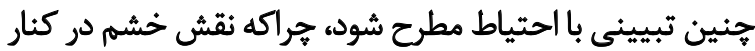

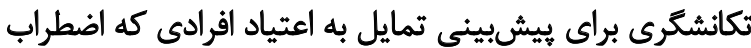

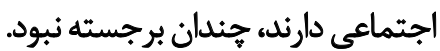

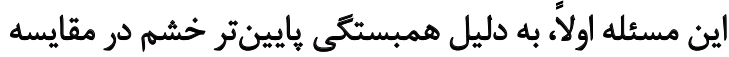

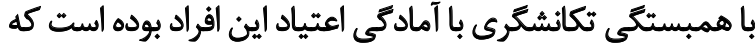

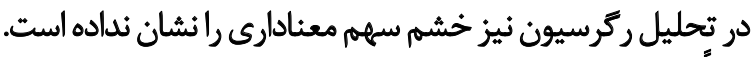

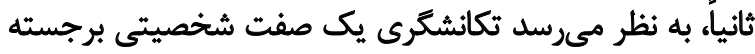

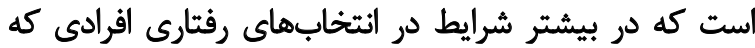

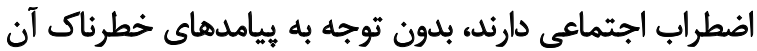

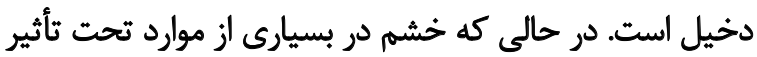

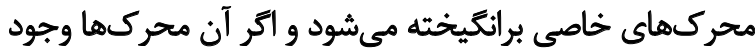

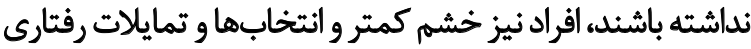

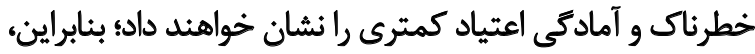

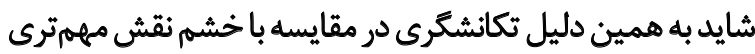

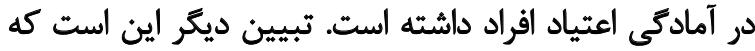

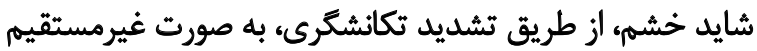

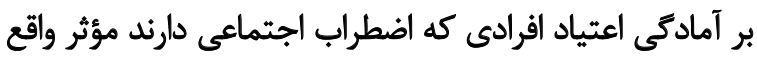

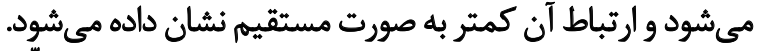

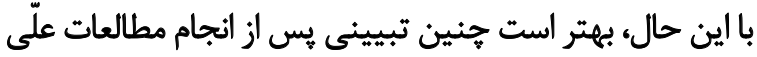

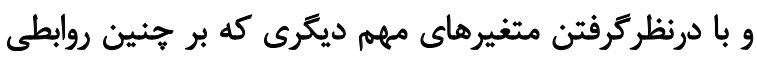
مؤثرند، به عنوان يك تبيين جدى تقين تقويت شوده. از محدوديتهاي عمده اين بثروهش، دشوارى در كنترل تمامى إئ

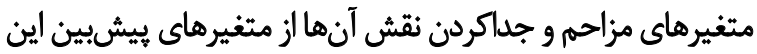

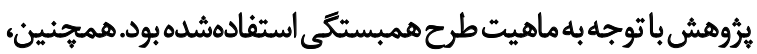

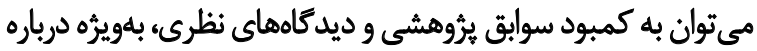

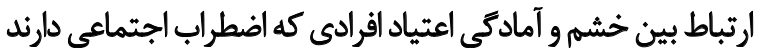

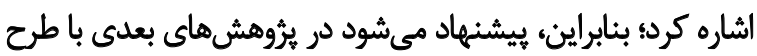

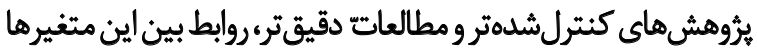

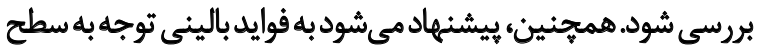

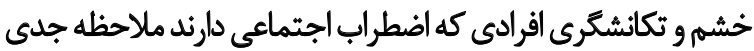

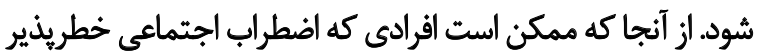

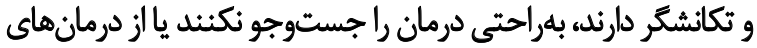

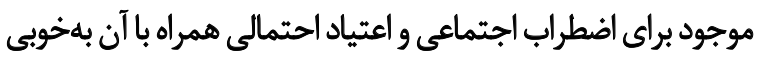

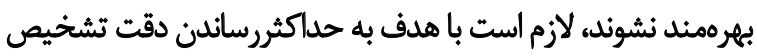

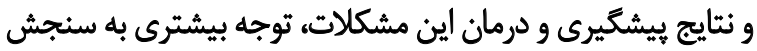
سطح خشم و تكانشكّرى آنها شود.
بازدارى رفتارى تحت تأثير قرار مى دهد (جاكوزكووياك وجتن

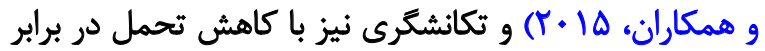

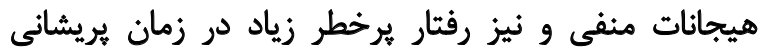

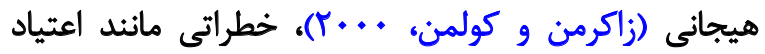

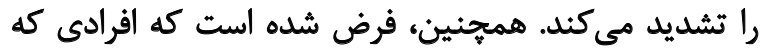

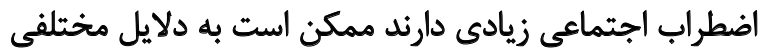

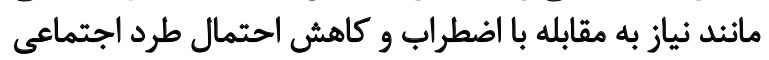

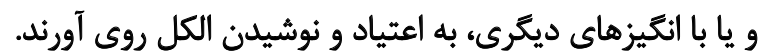
با اين حال، فرض درستتر و محتملتر اين است كه اين

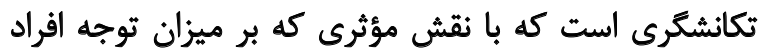

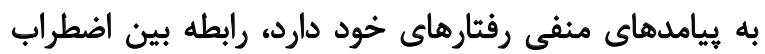

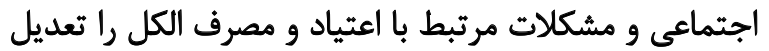

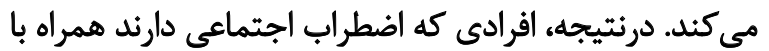

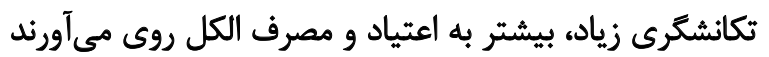

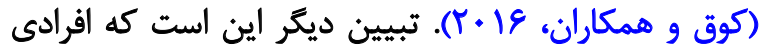

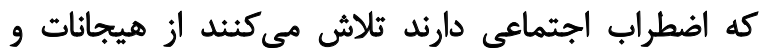

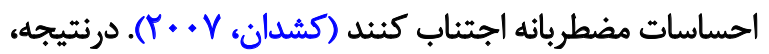

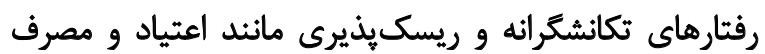

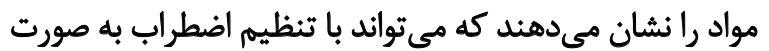

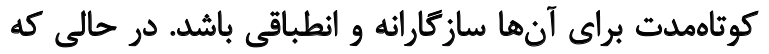

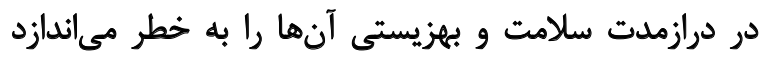

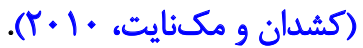
همجنين، از آنجا كه تعداد زيادى از افرادى كه اضطراب

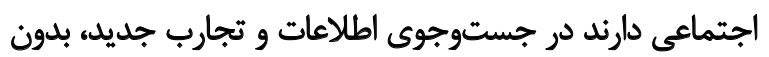

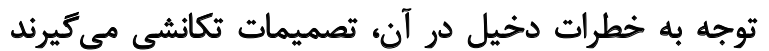

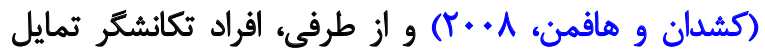

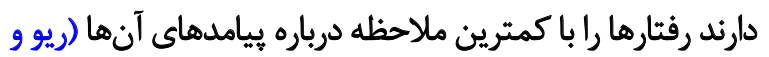

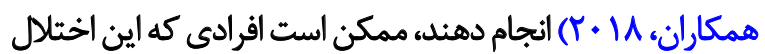
را دارند نسبت به رفتارهاى يرخطرى مانئد اعتياد مستعد باشند.

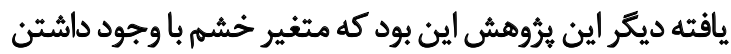

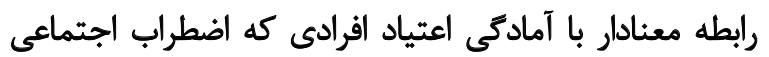

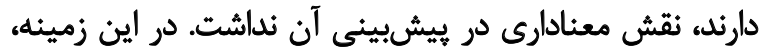

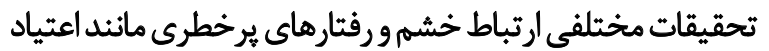

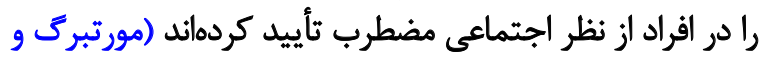

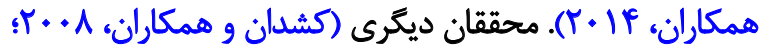

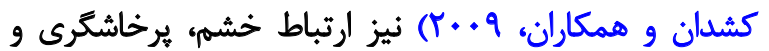

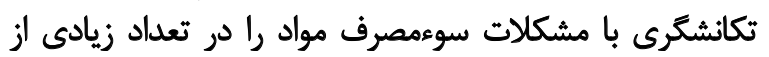

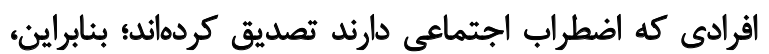

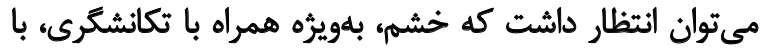

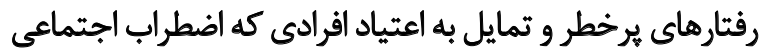
دارند مرتبط باشد. درباره ارتباط خشم و آمادگى اعتياد افرادى كه اضطراب 


\section{ملاحظات اخلاقى}

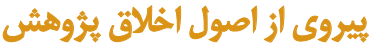

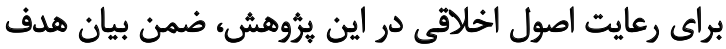

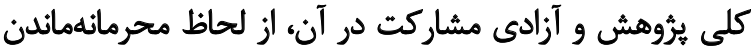

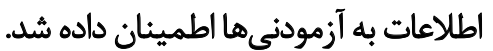

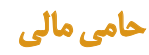

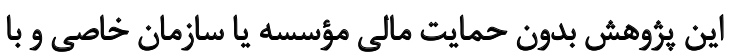
هزينه شخصى نويسنده در دانشعاه اراك انجام شده است است خان.

$$
\text { سباسكزازى }
$$

از تمامى كسانى كه محقق رادر انجام اين مطالعه يارى كردند،

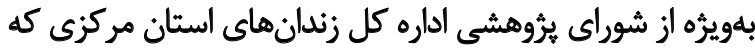

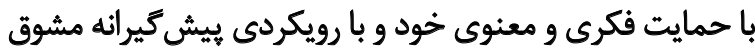
انجام جنين مطالعهاي بوده است، سياسكزارم. 
References

American Psychiatric Association. (2015). [Diagnostic and statistical manual of mental disorders (Persian)] [H. Hashemi Minaabad, D. Arab Ghahghahestani, Persian trans.]. Tehran: Roshd.

Ayduk, O., Mendoza-Denton, R., Mischel, W., Downey, G., Peake, P. K., \& Rodriguez, M. (2000). Regulating the interpersonal self: Strategic selfregulation for coping with rejection sensitivity. Journal of Personality and Social Psychology, 79(5), 776-92. [DOI:10.1037/0022-3514.79.5.776] [PMID]

Connor, K. M., Davidson, J. R. T., Churchill, L. E., Sherwood, A., Foa, E., \& Weisler, R. H. (2000). Psychometric properties of the Social Phobia Inventory (SPIN): New self-rating scale. British Journal of Psychiatry, 176(4), 379-86. [DOI:10.1192/bjp.176.4.379] [PMID]

Cremers, H. R., \& Roelofs, K. (2016). Social anxiety disorder: A critical overview of neurocognitive research. Wiley Interdisciplinary Reviews: Cognitive Science, 7(4), 218-32. [DOI:10.1002/wcs.1390] [PMID]

Curry, L. A., \& Youngblade, L. M. (2006). Negative affect, risk perception, and adolescent risk behavior. Journal of Applied Developmental Psychology, 27(5), 468-85. [DOI:10.1016/j.appdev.2006.06.001]

Dalrymple KL, Galione J, Hrabosky J, Chelminski I, Young D, O'brien E, et al. (2011). Diagnosing social anxiety disorder in the presence of obesity: Implications for a proposed change in DSM-5. Depression and Anxiety, 28(5), 377-82. [DOI:10.1002/da.20794] [PMID]

DeWall, C. N., Buchner, J. D., Lambert, N. M., Cohen A. S., \& Fincham, F. D. (2010). Bracing for the worst, but behaving the best: Social anxiety, hostility, and behavioral aggression. Journal of Anxiety Disorders, 24(2), 260-8. [DOI:10.1016/j.janxdis.2009.12.002] [PMID]

Dickman, S. J. (1990). Functional and dysfunctional impulsivity: Personality and cognitive correlates. Journal of Personality and Social Psychology, 58(1), 95-102. [DOI:10.1037/0022-3514.58.1.95] [PMID]

Dixon, L. J., Tull, M. T,. Lee, A. A., Kimbrel, N. A., \& Gratz, K. L. (2017). The role of emotion-driven impulse control difficulties in the relation between social anxiety and aggression. Journal of Clinical Psychology, 73(6), 722-32. [DOI:10.1002/jclp.22372] [PMID]

Duran, S., Ergün, S., Tekir, O., Çalışkan, T., \& Karadaş, A. (2018). Anger and tolerance levels of the inmates in prison. Archives of Psychiatric Nursing, 32(1), 66-70. [DOI:10.1016/j.apnu.2017.09.014]

Ekhtiari, H., Safaei, H., Esmaeeli Djavid, G., Atefvahid, M. K., Edalati, H., \& Mokri, A. (2008). [Reliability and validity of persian versions of eysenck, barratt, dickman and zuckerman questionnaires in assessing risky and impulsive behaviors (Persian)]. Iran University of Medical Sciences, 14(3), 326-36

Erwin, B. A., Heimberg, R. G., Schneier, F. R., \& Liebowitz, M. R. (2003). Anger experience and expression in social anxiety disorder: Pretreatment profile and predictors of attrition and response to cognitive-behavioral treatment. Behavior Therapy, 34(3), 331-50. [DOI:10.1016/S00057894(03)80004-7]

Gilbert, P., \& Miles, J. N. V. (2000). Sensitivity to social put-down: Its relationship to perceptions of social rank, shame, social anxiety, depression, anger and self-other blame. Personality and Individual Differences, 29(4), 757-74. [DOI:10.1016/S0191-8869(99)00230-5]

Hassanvand Amouzadeh M. (2017). [Evaluation of validity and reliability of social phobia inventory among students in Payame Noor University of Lorestan (Persian)]. Journal of Fasa University of Medical Sciences, 7(2), 181-9.
Hornsveld, R. H. J., Muris, P., \& Kraaimaat, F. W. (2011). The novaco anger scale-provocation inventory (1994 Version) in Dutch forensic psychiatric patients. Psychological Assessment, 23(4), 937-44. [DOI:10.1037/ a0024018] [PMID]

Jakuszkowiak-Wojten, K., Landowski, J., Wiglusz, M. S., \& Cubała, W. J. (2015). Impulsivity and anxiety disorders: A critical review. Psychiatria Danubina, 27(1), 452-5

Kachin, K. E., Newman, M. G., \& Pincus, A. L. (2001). An interpersonal problem approach to the division of social phobia subtypes. Behavior Therapy, 32(3), 479-501. [DOI:10.1016/S0005-7894(01)80032-0]

Kartari, P., Karia, S., Desousa, A., \& Prajapati, N. (2015). Social anxiety and impulsivity amongst internet addicts and substance. Paripex-Indian Journal of Research, 4(2), 43-4.

Kashdan, T. B.\&McKnight, P.E. (2010). The darkerside of social anxiety: When aggressive impulsivity prevails over shy inhibition. Current Directions in Psychological Science, 19(1), 47-50. [DOI:10.1177/0963721409359280]

Kashdan, T. B. (2007). Social anxiety spectrum and diminished positive experiences: Theoretical synthesis and meta-analysis. Clinical Psychology Review, 27(3), 348-65. [DOI:10.1016/j.cpr.2006.12.003] [PMID]

Kashdan, T. B., \& Collins, R. L. (2010). Social anxiety and the experience of positive emotion and anger in everyday life: An ecological momentary assessment approach. Anxiety, Stress, \& Coping, 23(3), 259-72. [DOI:10.1080/10615800802641950] [PMID]

Kashdan, T. B., \& Hofmann, S. G. (2008). The high novelty seeking, impulsive subtype of generalized social anxiety disorder. Depression and Anxiety, 25(6), 535-41. [DOI:10.1002/da.20382] [PMID]

Kashdan, T. B., Elhai, J. D., \& Breen, W. E. (2008). Social anxiety and disinhibition: An analysis of curiosity and social rank appraisals, approachavoidance conflicts, and disruptive risk-taking behavior. Journal of Anxiety Disorders, 22(6), 925-39. [DOI:10.1016/j.janxdis.2007.09.009] [PMID] [PMCID]

Kashdan, T. B., McKnight, P. E., Richey, J. A., \& Hofmann, S. G. (2009). When social anxiety disorder co-exists with risk-prone, approach behavior: Investigating a neglected, meaningful subset of people in the national comorbidity survey-replication. Behaviour Research and Therapy, 47(7), 559-68. [DOI:10.1016/j.brat.2009.03.010] [PMID] [PMCID]

Keil, V., Asbrand, J., TuschenCaffier, B., \& Schmitz, J. (2017). Children with social anxiety and other anxiety disorders show similar deficits in habitual emotional regulation: Evidence for a transdiagnostic phenomenon. European Child and Adolescent Psychiatry, 26(7), 749-57. [DOI:10.1007/ s00787-017-0942-x] [PMID]

Keough, M. T., Badawi, G., Nitka, D., O'Connor, R. M., \& Stewart, S. H. (2016) Impulsivity increases risk for coping-motivated drinking in undergraduates with elevated social anxiety. Personality and Individual Differences, 88(1), 45-50. [DOI:10.1016/j.paid.2015.08.036] [PMID] [PMCID]

Lerner, J. S., \& Keltner, D. (2001). Fear, anger, and risk. Journal of Personality and Social Psychology, 81(1), 146-59. [DOI:10.1037/00223514.81.1.146] [PMID]

Malekpour, M., Zangeneh, S., \& Aghababaei, S. (2012). [A study of the psychometric properties of novaco anger questionnaire (short form) in Isfahan (Persian)]. Journal of Researches of Cognitive and Bihavioral Sciences, 2(1), 1-8.

Meortberg, E., Tillfors, M., Van Zalk, N. \& Kerr, M. (2014). An atypical anxious-impulsive pattern of social anxiety disorder in an adult clinical population. Scandinavian Journal of Psychology, 55(4), 350-6. [DOI:10.1111/ sjop.12117] [PMID] 
Moeller, S. B., Novaco, R. W., Heinola-Nielsen, V., \& Hougaard, H. (2016). Validation of the novaco anger scale- provocation inventory (Danish) with nonclinical, clinical, and offender samples. Assesment, 23(5), 62436. [DOI:10.1177/1073191115583713] [PMID]

Moscovitch, D., McCabe, R., Antony, M., Rocca, L., \& Swinson, R. (2008). Anger experience and expression across the anxiety disorders. Depression and Anxiety, 25(2), 107-13. [DOI:10.1002/da.20280]

Nicholls, J., Staiger, P. K., Williams, J. S., Richardson, B., \& Kambouropoulos, N. (2014). When social anxiety co-occurs with substance use: Does an impulsive social anxiety subtype explain this unexpected relationship? Psychiatry Research, 220(3), 909-14. [DOI:10.1016/j.psychres.2014.08.040] [PMID]

Novaco, R. W. (1994). Anger as a risk factor for violence among the mentally disordered. In J. Monahan \& H. J. Steadman (Eds.), Violence and Mental Disorder: Developments in Risk Assessment (pp. 21-61). Chicago: University of Chicago.

O’Toole, M. S., Zachariae, R., \& Mennin, D. S. (2017). Social anxiety and emotion regulation flexibility: Considering emotion intensity and type as contextual factors. Anxiety, Stress \& Copping, 30(6), 716-24. [DOI:10. 1080/10615806.2017.1346792] [PMID]

Rapee, R. M., \& Heimberg, R. G. (1997). A cognitive-behavioral model of anxiety in social phobia. Behaviour Research and Therapy, 35(8), 741-56. [DOI:10.1016/S0005-7967(97)00022-3]

Ryu, H., Lee. J. Y., Choi, A. Park, S., Kim, D. J., \& Choi, J. S. (2018). The relationship between impulsivity and internet gaming disorder in young adults: Mediating effects of interpersonal relationships and depression. Internatinal Journal of Environmental Research and Public Health, 15(3), 458-68. [DOI:10.3390/ijerph15030458]
Sadock, b. J., \& Sadock, V. A. (2007). Kaplan Sadock's synopsis of psychiatry behavioral sciences (Persian)] [F. Rezaei, Persian trans.]. Tehran: Arjmand.

She, S., Eimontaite, I., Zhang, D., \& Sun, Y. (2017). Fear, anger, and risk preference reversals: An experimental study on a Chinese sample. Frontiers in Psychology, 8, 1371. [DOI:10.3389/fpsyg.2017.01371] [PMID] [PMCID]

Weed, N. C., \& Butcher, J. N. (1992). New measures for assessing alcohol and drug abuse with the MMPI-2: The APS and AAS. Journal of Personality Assessment, 58(2), 389-404. [DOI:10.1207/s15327752jpa5802_15] [PMID]

Zargar, Y. (2016). [Construction and validation of the Iranian scale of readiness for addiction (Persian)]. Paper presented at The second conference of the Iranian Psychological Association, Tehran, Iran, 25 February 2016.

Zargar, Y., Najarian, B., \& Naam, A. Z. (2009). [Relationship between personality characteristics (excitement, expression, psychological hardiness), religious attitude and marital satisfaction with drug addiction preparedness in employees of an industrial company in Ahwaz (Persian)]. Journal of Educational Sciences and Psychology, 15(1), 99-120.

Zuckerman, M., \& Kuhlman, M. D. (2000). Personality and risk-taking: Common biosocial factors. Journal of Personality, 68(6), 999-1029. [DOI:10.1111/1467-6494.00124] [PMID] 
This Page Intentionally Left Blank 